\title{
ON COMPOSITE ABSTRACT HOMOGENEOUS POLYNOMIALS ${ }^{1}$
}

\section{MORRIS MARDEN}

1. Introduction. In this note we study the nullsets of abstract homogeneous polynomials (a.h.p.) which are derived from given a.h.p.'s by certain kinds of composition.

Except when otherwise indicated, these polynomials are defined from $E$ to $K$ where $K$ is an algebraically closed field (of characteristic zero) and $E$ is a linear vector space over $K$. If $P_{n}$ denotes the family of all of a.h.p. of degree $n$, then by definition [1, pp. 760-763] $P \in P_{n}$ implies that for all $x, y \in E$ and $s, t \in K$,

$$
P(s x+t y)=\sum_{k=0}^{n} C_{n, k} A_{k}(x, y) s^{k} t^{n-k}
$$

where $C_{n, k}=n ! / k !(n-k) !$ and where the $A_{k}(x, y) \in K$, the $A_{k}$ being independent of $s$ and $t$. The $n$th polar of $P$ is defined from $E \times E$ $X \cdots \times E$ to $K$, as the form $P\left(x_{1}, x_{2}, \cdots, x_{n}\right)$ which is linear in each $x_{k}$, symmetric in the set $\left\{x_{k}\right\}$ and such that

$$
P(x, x, \cdots, x)=P(x)
$$

for all $x \in E$. In terms of the $n$th polar the coefficients $A_{k}$ of $P$ are given by the formulas

$$
A_{k}(x, y)=\left[\odot\left(x_{1}, \cdots, x_{n}\right): x_{j_{\S} k}=x, x_{>>k}=y\right] .
$$

If $P \in P_{n}$, the nullset $Z_{P}$ of $P$ corresponding to given $x, y \in E$, namely

$$
Z_{P}=\{s x+t y: P(s x+t y)=0, s x+t y \neq 0\},
$$

belongs to sets that we shall specify by inequalities involving Hermitian symmetric forms $H(x, y)$. These forms may be defined as in the complex plane, since we may write $K=K_{0}(i)$ where $K_{0}$ is a maximally ordered subfield of $K$ and $-i^{2}$ is the unit element in $K$ and since therefore with each $\kappa \in K$ we may associate a "conjugate" element $\bar{\kappa} \in K$. Thus $H(x, y)$ satisfies the three requirements: $H(x, y) \in K$ for all $x, y \in E, H(x, y)$ is linear in $x$ for any fixed $y$ and

$$
H(y, x)=\overline{H(x, y)} \text {. }
$$

Presented to the Society August 30, 1968; received by the editors September 26, 1968.

${ }^{1}$ Work on this paper was supported by the National Science Foundation under Grant GP-5516. 
The general aim of the present note is to describe the nullset of a composite a.h.p. $R$ relative to the nullsets of the a.h.p. from which $R$ was derived. The results obtained are analogous to some wellknown theorems on the zeros of composite polynomials in the complex plane.

2. An apolarity-like relation. To aid our study of the composites of two a.h.p. $P$ and $Q$ with $P$ given by (1.1) and $Q$ given similarly by

$$
Q(s x+t y)=\sum_{k=0}^{n} C_{n, k} B_{k}(x, y) x^{k} t^{n-k}
$$

we introduce an operator $\Phi(P, Q ; x, \xi)$ defined by the relation

$$
\begin{aligned}
& \Phi(P, Q ; s x+t y ; \sigma \xi+\tau \eta) \\
& \quad=\sum_{k=0}^{n}(-1)^{k} C_{n, k} A_{k}(x, y) B_{n-k}(\xi, \eta) s^{k} t^{n-k} \sigma^{n-k} \tau^{k} .
\end{aligned}
$$

So defined, $\Phi(P, Q ; x, \xi)$ is an $n$th degree a.h.p. in $x$ and in $\xi$. It is a linear functional of $P$ and of $Q$ that has the following properties for $x, y, \xi, \eta \in E ; s, t, \sigma, \tau \in K$ :

$$
\begin{aligned}
& \Phi(P, Q ; s x+t y ; \sigma \xi+\tau \eta) \in K, \\
& \Phi(Q, P ; \xi, x)=(-1)^{n} \Phi(P, Q ; x, \xi), \\
& \Phi(P, Q ; s x+t x ; \sigma \xi+\tau \eta)=P(x) Q(\sigma t \xi-\tau s \eta) .
\end{aligned}
$$

The relations (2.3) and (2.4) are obvious. To deduce (2.5), we note that, since $P \in P_{n}, P(s x+t x)=(s+t)^{n} P(x)$ and thus $A_{k}(x, x)=P(x)$, for all $x \in E$ and $k=0,1, \cdots, n$. Hence, from (2.2)

$$
\begin{aligned}
\Phi(P, Q ; s x+t x ; \sigma \xi+\tau \eta) & =P(x) \sum_{k=0}^{n}(-1)^{k} C_{n, k} B_{n-k}(\xi, \eta) s^{k} t^{n-k} \sigma^{n-k} \tau^{k} \\
& =P(x) Q(\sigma t \xi-\tau s \eta) .
\end{aligned}
$$

In particular, we learn from (2.5) that, for all $x, \xi \in E$,

$$
\Phi(P, Q ; s x+t x ; s \xi+t \xi)=0 .
$$

The operator $\Phi$ has another property which we now state using the assumption that $K$ is algebraically closed and that hence we may write $Q$ in the form

$$
Q(s \xi+t \eta)=\prod_{j=1}^{n}\left(\delta_{j} s-\gamma_{j} t\right),
$$

where $\delta_{j} \equiv \delta_{j}(\xi, \eta) \in K$ and $\gamma_{j} \equiv \gamma_{j}(\xi, \eta) \in K$ for $\xi, \eta \in E$. 
THEOREM (2.1). Let $P, Q \in P_{n}$ with $Q(x)$ written in form (2.7). Then for any $\xi, \eta \in E$

$$
\Phi(P, Q ; \xi+\eta ; \xi+\eta)=\odot\left(\gamma_{1} \xi+\delta_{1} \eta, \gamma_{2} \xi+\delta_{2} \eta, \cdots, \gamma_{n} \xi+\delta_{n} \eta\right) .
$$

Thus, if Theorem (2.1) is valid, also

$(2.8)^{\prime} \Phi(P, Q ; s x+t y, s x+t y)=\rho\left(s \gamma_{1} x+t \delta_{1} y, \cdots, s \gamma_{n} x+t \delta_{n} y\right)$.

By (2.4), if $P(s x+t y)=\prod_{1}^{n}\left(\beta_{k} s-\alpha_{k} t\right)$, $(2.8)^{\prime \prime} \Phi(P, Q ; s x+t y, s x+t y)=(-1)^{n} Q\left(s \alpha_{1} x+t \beta_{1} y, \cdots, s \alpha_{n} x+t \beta_{n} y\right)$.

Proof. From (2.1) and (2.7), follows that

$$
C_{n, k} B_{k}(\xi, \eta)=(-1)^{n-k} S_{k}
$$

where $S_{k}$ is the sum of all products obtained from $\left[\delta_{1} \delta_{2} \cdots \delta_{k} \gamma_{k+1} \gamma_{k+2}\right.$ $\left.\cdots \gamma_{n}\right]$ by permuting the subscripts $1,2, \cdots, n$ in all possible ways. On the other hand, using the fact that $\rho\left(x_{1}, x_{2}, \cdots, x_{n}\right)$ is linear in each $x_{n}$, we find that

$$
\begin{aligned}
\mathcal{P}\left(\gamma_{1} \xi+\delta_{1} \eta,\right. & \left.\cdots, \gamma_{n} \xi+\delta_{n} \eta\right) \\
& =\sum_{k=0}^{n} S_{n-k}\left[\odot\left(x_{1}, \cdots, x_{n}\right): x_{j \leq k}=\xi, x_{j>k}=\eta\right] \\
& =\sum_{k=0}^{n}(-1)^{k} C_{n, k} B_{n-k}(\xi, \eta) A_{k}(\xi, \eta)=\Phi(P, Q ; \xi+\eta ; \xi+\eta) .
\end{aligned}
$$

The last line follows from (2.9) and (1.3).

3. Theorems on composite a.h.p. We now prove a number of theorems on the nullsets of composite a.h.p. The first is analogous to a corollary of Grace's theorem [2, pp. 62-63], cf. also [3].

TheOREm (3.1). Let $H(x, y)$ be a Hermitian symmetric form and let

$$
E_{1}=\{x \in E: H(x, x) \leqq 0, x \neq 0\} .
$$

Let $P, Q \in P_{n}$ and let $Z_{P}$ and $Z_{Q}$ be the nullsets of $P$ and $Q$ respectively corresponding to given $\xi, \eta \in E$. If $Z_{P} \subset E_{1}$ and if $Q$ be such that

$$
\Phi(P, Q ; \xi+\eta ; \xi+\eta)=0,
$$

then

$$
Z_{Q} \cap E_{1} \neq \varnothing \text {. }
$$

PROOF. If on the contrary $Z_{Q} \cap E_{1}=\varnothing$, then $Z_{Q} \subset E-E_{1}$. That is, writing $Q(x)$ as in (2.7) with the given $\xi$, $\eta$, we conclude that

$$
\gamma_{k} \xi+\delta_{k} \eta \in E-E_{1} \quad \text { for } k=1,2, \cdots, n \text {. }
$$


We may now apply the following theorem due to Hörmander [3]: If $P(x) \neq 0$ for all $x \in E-E_{1}$, then also $\odot\left(x_{1}, x_{2}, \cdots, x_{n}\right) \neq 0$ for all $x_{k} \in E-E_{1}$. We conclude that

$$
P\left(\gamma_{1} \xi+\delta_{1} \eta, \gamma_{2} \xi+\delta_{2} \eta, \cdots, \gamma_{n} \xi+\delta_{n} \eta\right) \neq 0,
$$

a result which contradicts (3.2) in view of Theorem (2.1). Thus, Theorem (3.1) has been established.

We next develop a theorem analogous to a composition theorem in the complex plane due to Szegö [2, pp. 65-66], namely: Let $P(z)$ $=\sum_{0}^{n} C_{n, k} A_{k} z^{k}, Q(z)=\sum_{0}^{n} C_{n, k} B_{k} z^{k}, R(z)=\sum_{0}^{n} C_{n, k} A_{k} B_{k} z^{k}$. Let $\Gamma$ be any circular region containing the zeros of $P$. Then every zero of $R$ has the form $(-\beta \gamma)$ where $\beta$ is a zero of $Q$ and $\gamma \in \Gamma$. A counterpart in vector space is the following:

Theorem (3.2). Let $P, Q \in P_{n}$ and $R \in P_{2 n}$ be defined by (1.1) and (2.1) and

$$
R(s x+t y)=\sum_{k=0}^{n}(-1)^{k} C_{n, k} A_{k}(x, y) B_{k}(x, y) s^{2 k} t^{2(n-k)} .
$$

Corresponding to given $x, y \in E$ such that $Q(x) Q(y) \neq 0$, let $Z_{P}, Z_{Q}$ and $Z_{R}$ be the nullsets of $P, Q$ and $R$ respectively. Let $E_{1}$ be defined by (3.1). If $Z_{P} \subset E_{1}$ and if $(\mu x+\nu y) \in Z_{R}$, then $\left(\mu^{2} x+\nu^{2} y\right)$ belongs to the set $\{\alpha \gamma x+\beta \delta y\}$ for all $\alpha, \beta, \gamma, \delta \in K$ such that

$$
\alpha x+\beta y \in E_{1}, \quad \gamma x+\delta y \in Z_{Q} .
$$

Proof. Let $(\mu x+\nu y)$ be any zero of $R$. That is,

$$
R(\mu x+\nu y)=\sum_{k=0}^{n}(-1)^{k} C_{n, k} A_{k}(x, y) B_{k}(x, y) \mu^{2 k} \nu^{2(n-k)}=0 .
$$

Writing $Q$ as in (2.1) and (2.7) and noting that $\gamma_{1} \gamma_{2} \cdots \gamma_{n} \delta_{1} \delta_{2} \cdots \delta_{n}=(-1)^{n} B_{n}(x, y) B_{0}(x, y)=(-1)^{n} Q(x) Q(y) \neq 0$, we define $Q^{*} \in P_{n}$ by the relations

$$
\begin{aligned}
Q^{*}(s x+t y) & =\sum_{k=0}^{n} C_{n, k} B_{k}^{*}(x, y) s^{k} t^{n-k} \\
& =\prod_{k=1}^{n}\left(\delta_{k}^{*} s-\nu_{k}^{*} t\right)
\end{aligned}
$$

with $\delta_{k}^{*}=\nu^{2} \delta_{k}^{-1}$ and $\gamma_{k}^{*}=\mu^{2} \gamma^{-1}$. Using notation similar to (2.9), we find

$$
\delta_{1} \delta_{2} \cdots \delta_{n} \gamma_{1} \gamma_{2} \cdots \gamma_{n} S_{k}^{*}=\nu^{2 k} \mu^{2(n-k)} S_{n-k}
$$


Hence

$$
B_{0}(x, y) B_{n}(x, y) B_{k}^{*}(x, y)=\nu^{2 k} \mu^{2(n-k)} B_{n-k}(x, y) .
$$

We may now write equation (3.5) as

Hence,

$$
R(\mu x+\nu y)=B_{0}(x, y) B_{n}(x, y) \sum_{k=0}^{n}(-1)^{k} C_{n, k} A_{k}(x, y) B_{n-k}^{*}(x, y)=0 .
$$

$$
\Phi\left(P, Q^{*} ; x+y, x+y\right)=0 .
$$

From Theorem (3.1), we now infer that at least one zero $\alpha x+\beta y$ of $Q^{*}$ lies on $E_{1}$. From (3.6), for some value of $k$

$$
\alpha=\gamma_{k}^{*}=\mu^{2} \gamma_{k}^{-1}, \quad \beta=\delta_{k}^{*}=\nu^{2} \delta_{k}^{-1} .
$$

That is, if $\mu x+\nu y \in Z_{R}$, then

$$
\mu^{2} x+\nu^{2} y \in\left\{\alpha \gamma x+\beta \delta y: \alpha x+\beta y \in E_{1}, \gamma x+\delta y \in Z_{Q}\right\} .
$$

This completes the proof of Theorem (3.2).

4. Polynomials on vectors to vectors. So far we have considered a.h.p. which assume values in a field $K$. We now extend Theorem (3.1) to a.h.p. which assume values on a supportable subset of a vector space $G$. A subset $M$ of $G$ is said to be supportable if to every $\zeta \in G-M$ there corresponds a linear form $L(w)$ such that $L(\zeta)=0$ but $L(w) \neq 0$ for $w \in M$. (See [3].)

Theorem (4.1). Let $E$ and $G$ be vector spaces over $K$ and let $M$ be a supportable subset of $G$. Let $P$ and $Q$ be a.h.p. defined on $E$ to $G$. Corresponding to given $\xi, \eta \in E$, let

$$
\begin{aligned}
& E_{P}=\{s \xi+t \eta \in E: P(s \xi+t \eta) \in M\}, \\
& E_{Q}=\{s \xi+t \eta \in E: Q(s \xi+t \eta) \in M\} .
\end{aligned}
$$

Assume $E_{P}=E-E_{1}$ where $E_{1}$ is defined by (3.1). For some $\zeta_{0} \in G-M$ and the corresponding linear form $L_{0}(w)$, let $Q$ be such that

$$
\Phi\left(L_{0}(P), L_{0}(Q) ; \xi+\eta, \xi+\eta\right)=0 .
$$

Then

$$
\left(E-E_{P}\right) \cap\left(E-E_{Q}\right) \neq \varnothing .
$$

REMark. Theorem (4.1) is trivial if either $E_{P}=\varnothing$ or $E_{Q}=\varnothing$.

ProOF. For every $\zeta \in G-M$ and its corresponding $L(w)$, both $L(P(x))$ and $L(Q(x))$ are a.h.p. of degree $n$ and

$$
L(P(x)) \neq 0 \quad \text { for } x \in E_{P}, \quad L(Q(x)) \neq 0 \text { for } x \in E_{Q} .
$$


Hence, $Z_{L(P)} \subset E-E_{P}, Z_{L(Q)} \subset E-E_{Q}$. Since (4.1) holds, Theorem (3.1) implies (4.2), as was to be proved.

\section{REFERENCES}

1. E. Hille and R. S. Phillips, Functional analysis and semigroups, Amer. Math. Soc. Colloq. Publ., Vol. 31, Amer. Math. Soc., Providence, R. I., 1957.

2. M. Marden, Geometry of polynomials, Math. Surveys No. 3, Amer. Math. Soc., Providence, R. I., 1966.

3. L. Hörmander, On a theorem of Grace, Math. Scand. 2 (1954), 55-64.

4. M. Marden, $A$ generalization of a theorem of Bôcher, SIAM J. Numer. Anal. (Walsh Jubilee Issue) 3 (1966), 269-275.

UNIVERSITY OF WisCoNSIN-MilWAUKEE 